ORIGINAL ARTICLE

\title{
Eccentric training in patients with chronic Achilles tendinosis: normalised tendon structure and decreased thickness at follow up
}

\author{
L Öhberg, R Lorentzon, H Alfredson
}

Br J Sports Med 2004;38:8-11. doi: 10.1136/bjsm.2001.000284

See end of article for authors' affiliations

......................

Correspondence to: Dr Öhberg, Department of Radiology, University Hospital of Umeå, 90185 Umeå, Sweden;

lars.ohberg.us@vll.se

Accepted

23 December 2002

\begin{abstract}
Objective: To prospectively investigate tendon thickness and tendon structure by ultrasonography in patients treated with eccentric calf muscle training for painful chronic Achilles tendinosis located at the 2$6 \mathrm{~cm}$ level in the tendon.

Methods: The patients were examined with grey scale ultrasonography before and 3.8 years (mean) after the 12 week eccentric training regimen. At follow up, a questionnaire assessed present activity level and satisfaction with treatment.

Results: Twenty six tendons in twenty five patients (19 men and six women) with a mean age of 50 years were followed for a mean of 3.8 years (range 1.6-7.75). All patients had a long duration of painful symptoms (mean 17.1 months) from chronic Achilles tendinosis before treatment. At follow up, 22 of 25 patients were satisfied with treatment and active in Achilles tendon loading activities at the desired level. Ultrasonography showed that tendon thickness (at the widest part) had decreased significantly $(p<0.005)$ after treatment $(7.6(2.3)$ v 8.8 (3) $\mathrm{mm}$; mean (SD)). In untreated normal tendons, there was no significant difference in thickness after treatment $(5.3(1.3) \mathrm{mm}$ before and $5.9(0.8) \mathrm{mm}$ after). All tendons with tendinosis had structural abnormalities (hypoechoic areas and irregular structure) before the start of treatment. After treatment, the structure was normal in 19 of the 26 tendons. Six of the seven patients with remaining structural abnormalities experienced pain in the tendon during loading.

Conclusions: Ultrasonographic follow up of patients with mid-portion painful chronic Achilles tendinosis treated with eccentric calf muscle training showed a localised decrease in tendon thickness and a normalised tendon structure in most patients. Remaining structural tendon abnormalities seemed to be associated with residual pain in the tendon.
\end{abstract}

35-73) and a long duration (mean 17.1 months) of painful symptoms from the mid-portion $(2-6 \mathrm{~cm}$ level) of the Achilles tendon, were included in this investigation. The condition was considered to be in the chronic stage. None of the patients had received surgical treatment.

In all patients there was thickening to various extents, which was painful during palpation and activity, located at the $2-6 \mathrm{~cm}$ level from the calcaneal insertion. Ultrasonography showed localised thickening of the tendon, focal hypoechoic areas, and irregular tendon structure, corresponding to the painful area. All ultrasonographic examinations at inclusion and follow up were performed by the same experienced musculoskeletal radiologist (LÖ).

All patients were fully informed and gave their consent to participate in the study. The study was approved by the ethical committee at the Medical Faculty of the University of Umeå.

\section{Drop outs}

Thirty five patients ( 36 tendons) were asked to participate in the investigation. Ten declined because of little interest and a long distance to travel. All stated that they were satisfied with the results of treatment and active in Achilles tendon loading activities at the desired level.

\section{Grey scale ultrasonography}

Ultrasonography was performed with a linear transducer (Acuson Sequoia 512) with 8-13 MHz frequency before and after the eccentric training regimen. The examinations were carried out with the subject prone with both feet hanging over the end of the examination table to allow their 
movement. The Achilles tendons were examined in longitudinal and transverse plane. It was important to examine the tendons parallel, with the fibres in the longitudinal plane and perpendicular in the transverse plane to minimise artefacts. Both tendons were always examined in the same manner.

All examinations were saved on a magnetic optic disc (MOdisc), and all measurements were repeated. The test to test reliability for the observer (LÖ) was evaluated from 10 examinations of one tendon during a short time, with repositioning between investigations. The coefficient of variation was estimated to be $1.1 \%$.

\section{Questionnaire}

At follow up (mean 3.8 years), all patients answered a questionnaire assessing their satisfaction with the result of treatment with eccentric calf muscle training, present tendon loading activity level, and tendon symptoms.

\section{Statistical evaluation}

The Wilcoxon signed rank test was used to study the differences in tendon thickness before and after treatment.

\section{RESULTS}

Table 1 shows the basic characteristics and activity levels of the patients.

Three patients were not satisfied with the results of treatment and had to change to an activity with a lower level of Achilles tendon loading because of pain in the tendon during activity. One changed from floor-ball to walking, one from floor-ball to jogging, and the third from jogging to cross country skiing.

Table 2 shows tendon thickness (widest part) and the occurrence of structural abnormalities in the tendon before and after treatment, tendon symptoms (pain, stiffness) after treatment, and follow up time.

In all patients with painful thickening at the $2-6 \mathrm{~cm}$ level in the tendon, localised widening, focal hypoechoic areas, and irregular structure, corresponding to the painful area, were seen before treatment (fig 1). Follow up was performed a mean of 3.8 years (range 1.6-7.75) after treatment with eccentric calf muscle training. There was a significant $(p<0.005)$ decrease in tendon thickness (at the widest part) between the measurements before and after treatment ( 8.8 (3) $\mathrm{mm}$ and $7.6(2.3) \mathrm{mm}$ (mean (SD)) respectively).

Table 1 Basic data on the 25 patients with chronic Achilles tendinosis

\begin{tabular}{ll}
\hline Variable & Number or value \\
\hline Sport before treatment & 11 \\
Jogging & 7 \\
Walking & 3 \\
Floor-ball & 2 \\
Skiing* & 1 \\
Badminton & 1 \\
Cycling & 11 \\
Sport after treatment & 8 \\
Jogging & 1 \\
Walking & 3 \\
Floor-ball & 1 \\
Skiing* & 1 \\
Badminton & 17.1 (mean) (range 6-120) \\
Cycling & $50.4(9.6)$ \\
Duration of symptoms (months) & 173.6 (7.1) \\
Age (years) & 80.5 (12.3) \\
Height (cm) & \\
Weight (kg) & \\
\hline *Cross country skiing. &
\end{tabular}

Table 2 Details on the tendons of 25 patients with Achilles tendinosis

\begin{tabular}{|c|c|c|c|c|c|}
\hline \multirow[b]{2}{*}{ Sex/age } & \multicolumn{2}{|c|}{ Thickness (mm) } & \multicolumn{2}{|c|}{ Structure } & \multirow{2}{*}{$\begin{array}{l}\text { Follow up } \\
\text { (years) }\end{array}$} \\
\hline & Before & After & Before & After & \\
\hline Female/58 & 11.0 & 11.5 & $x$ & $x$ & 3.75 \\
\hline Male/54 & 4.0 & 6.4 & $x$ & 0 & 4.25 \\
\hline Male/50 & 6.3 & 6.1 & $x$ & 0 & 5.7 \\
\hline Female/51 & 12.0 & 10.0 & $x$ & $x \dagger \ddagger$ & 4.5 \\
\hline Male/45 & 18.2 & 14.7 & $x$ & $x \dagger$ & 3.9 \\
\hline Male/54 & 9.2 & 7.3 & $x$ & 0 & 4.9 \\
\hline Female/59 & 7.5 & 6.1 & $x$ & 0 & 7.75 \\
\hline Male/47 & 7.1 & 6.4 & $\hat{x}$ & 0 & 4.0 \\
\hline Male/51 & 7.2 & 5.7 & $x$ & 0 & 5.2 \\
\hline Male/41 & 6.7 & 6.5 & $\mathrm{x}$ & 0 & 4.3 \\
\hline Male/47 & 14.2 & 13.2 & $\hat{x}$ & $x^{*} \ddagger$ & 5.8 \\
\hline Male/55 & 7.3 & 7.5 & $x$ & $x \dagger$ & 1.9 \\
\hline Male/47 & 9.1 & 7.4 & $x$ & 0 & 3.6 \\
\hline Male/50 & 9.5 & 7.2 & $x$ & 0 & 4.0 \\
\hline Female/56 & 5.6 & 6.3 & $\mathrm{x}$ & 0 & 3.5 \\
\hline Male/55 & 8.7 & 6.0 & $x$ & 0† & 6.6 \\
\hline Male/45 & 6.7 & 7.3 & $x$ & $x+$ & 4.2 \\
\hline Female/62 & 10.2 & 7.0 & $\hat{x}$ & $\hat{0 † \ddagger}$ & 2.8 \\
\hline Male/36 & 6.6 & 5.3 & $x$ & $0 * \pm$ & 1.6 \\
\hline Male/36 & 6.8 & 5.3 & $x$ & $0^{+}$ & 1.6 \\
\hline Male/56 & 11.2 & 9.0 & $x$ & 0 & 1.9 \\
\hline Female/54 & 8.6 & 6.6 & $\hat{x}$ & 0 & 1.8 \\
\hline Male/47 & 6.1 & 6.3 & $x$ & 0 & 1.75 \\
\hline Male/63 & 10.3 & 7.2 & $x$ & 0 & 1.8 \\
\hline Male/57 & 7.0 & 7.2 & $\mathrm{x}$ & $x^{*} \ddagger$ & 1.6 \\
\hline Male/73 & 12.0 & 8.0 & $x$ & 0 & 1.8 \\
\hline
\end{tabular}

Tendon thickness (widest part) and the occurrence of structural abnormalities in the tendon (graded $\mathrm{x}$ ), before and after treatment with eccentric calf muscle training, in 25 patients with painful chronic Achilles tendinosis in 26 tendons.

*Not satisfied with the result of treatment, could not be active at the desired activity level because of pain in the Achilles tendon. One patient changed from floor-ball to jogging, one from floor-ball to walking, and one from jogging to cross country skiing

†Satisfied with the result of treatment and able to perform Achilles tendon loading activities at the desired level. There was some remaining pain in the tendon, but the pain did not prevent participation in heavy Achilles tendon loading activities.

†Experienced tendon stiffness after rest (morning stiffness).

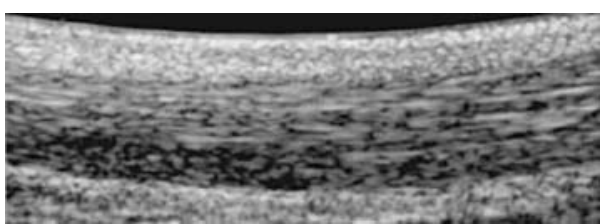

Figure 1 An Achilles tendon with chronic tendinosis shown by ultrasonography before treatment with eccentric calf muscle training. The typical findings are a localised thickening, focal hypoechoic areas, and an irregular tendon fibre structure.

In 13 normal tendons there was a normal tendon thickness before treatment, and there was no significant difference in tendon thickness between the measurements before and after treatment $(5.3$ (1.3) and 5.9 (0.8) mm respectively; mean (SD)).

Before treatment, there was an abnormal structure (hypoechoic areas and irregular structure) in all tendons from patients with painful chronic Achilles tendinosis. After treatment, the structure was normal in 19 of the 26 tendons (fig 2). Two patients with remaining structural tendon abnormalities after treatment were not satisfied with the result of treatment because of residual pain in the Achilles tendon during heavy tendon loading activities. These two patients had a follow up time of 5.8 and 1.6 years. Four patients with remaining structural abnormalities were 


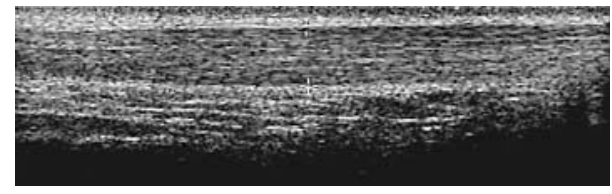

Figure 2 Ultrasonographic image of an Achilles tendon after treatment with eccentric calf muscle training. The typical findings are a decrease in tendon thickness, no hypoechoic areas, and a regular tendon fibre structure.

satisfied with the result of treatment, but had some residual pain in the tendon during heavy Achilles tendon loading activities. The follow up time for these four patients was 1.94.5 years. Five patients with remaining pain after treatment also had residual morning stiffness in the tendon.

One patient with remaining structural tendon abnormalities after treatment was satisfied with the result of treatment and had no painful symptoms or morning stiffness after treatment (follow up time 3.75 years). One patient with residual morning stiffness in the tendon had a normal tendon structure and no pain in the tendon after treatment.

One patient who was not satisfied with the result of treatment had a normal structure but remaining pain and morning stiffness in the tendon after treatment (follow up time 1.6 years $)$.

\section{DISCUSSION}

This prospective investigation, using ultrasonography, shows that, after treatment of painful mid-portion chronic Achilles tendinosis with eccentric calf muscle training, in most successfully treated tendons there was decreased tendon thickness and a normalised tendon structure in the area with tendinosis.

In patients with chronic mid-portion Achilles tendon pain, the localised widening of the Achilles tendon and focal hypoechoic areas on ultrasonography ${ }^{6}{ }^{12}$ have been shown to be caused by increased amounts of interfibrillar glycosaminoglycans, changes in the collagen structure and arrangement, and, in $65 \%$ of the cases, increased vascularity. ${ }^{6}$ The name of this condition is nowadays generally accepted to be chronic Achilles tendinosis. ${ }^{5}{ }^{15}$ It has generally been considered to be difficult to treat, but recently good short term clinical results have been achieved with a treatment model consisting of painful eccentric calf muscle training. ${ }^{8}{ }^{9}$ In our study, the good results have persisted, with 22 of 25 patients being satisfied with the result of treatment at a 3.8 year (mean) follow up.

The background to the good clinical results achieved with eccentric training is not known, and the possible effects of this treatment regimen on tendon thickness and tendon structure have not previously been evaluated. Grey scale ultrasonography is commonly used to investigate Achilles tendon injuries, and has been shown to be a reliable and cost effective method of identifying abnormalities, such as increased tendon thickness, hypoechoic areas, and irregular structure. ${ }^{61-13}$ Therefore we decided to use ultrasonography to study tendon thickness and tendon structure before and after treatment with eccentric training. It is generally agreed that the reliability of ultrasonographic examinations increases with the experience of the operator (radiologist), and in our study the same highly experienced radiologist performed all examinations.

We found that tendon width had decreased significantly 3.8 years after eccentric calf muscle training. Furthermore, before treatment all patients had hypoechoic areas and an irregular tendon structure, but at follow up the tendon structure was normal (no hypoechoic areas and regular structure) in 19 of the 26 tendons. We found no indications that the length of the follow up was important - that is, that the tendons with remaining structural abnormalities were the ones with the shortest follow up periods. Interestingly, of the seven patients with remaining structural abnormalities, all but one also had residual pain during tendon loading activity. These findings indicate that there may be an association between the ultrasonographic findings of hypoechoic areas/irregular tendon structure and Achilles tendon pain during tendon loading activity.

We cannot explain why tendon thickness decreased and tendon structure was normalised in most of the successfully treated patients. Furthermore, we do not know at what point during or after treatment this positive effect occurred. Ideally, serial ultrasonographic follow ups should have been performed throughout the treatment and follow up period, but that was impractical. Furthermore, for ethical reasons we have no control group; to have allowed a group of patients with symptoms of long duration to remain without treatment for up to five years would have been ethically wrong. It would seem that the eccentric training induced remodelling of the injured tendon. It has been shown that areas with tendinosis contain high concentrations of glycosaminoglycans, irregular fibre structure and arrangement, and vessel ingrowth of unknown cause and pathogenesis. ${ }^{6}$ The eccentric training regimen may induce a response that normalises the concentrations of glycosaminoglycans and possibly also enables normalisation of the fibre arrangement, resulting in decreased tendon thickness. In our experience, in most patients, the pain in the tendon diminishes and then disappears during the 12 week training period. It may be that the pain recedes during the early part of a possible remodelling phase, but normalisation of tendon thickness and structure may be more time consuming.

In a recent study showing that patients with chronic painful Achilles tendinosis had vascular ingrowth (neovascularisation) in the area with tendinosis, we showed that, during eccentric loading, the blood flow in the area with neovascularisation stopped. ${ }^{16}$ Therefore the decrease in tendon thickness may be due to reduced neovascularisation in the area with tendinosis, induced by the eccentric training.

In conclusion, eccentric calf muscle training in patients with painful mid-portion chronic Achilles tendinosis has good short and mid term clinical results. At ultrasonographic follow up, in most patients, tendon thickness is decreased and the structure is normalised. Structural abnormalities that remain at follow up seem to be associated with residual pain in the tendon.

\section{Authors' affiliations}

L Öhberg, Department of Radiation Sciences, Diagnostic Radiology, Umeå University, Umeå, Sweden

R Lorentzon, $\mathbf{H}$ Alfredson, Department of Surgical and Perioperative Science, Sports Medicine and National Institute for Working Life, University of Umeå

\section{Take home message}

Painful eccentric calf muscle training has good short and mid term clinical results in patients with chronic painful midportion tendinosis. In successfully treated patients, ultrasonographic follow up shows decreased thickness and normalised structure in the affected area. There may be a relation between residual pain in the tendon and remaining structural abnormalities. 


\section{REFERENCES}

1 Kvist M. Achilles tendon injuries in athletes. Sports Med 1994;18:173-201.

2 Józsa L, Kannus P. Human tendons. Anatomy, physiology, and pathology. Champaign, IL: Human Kinetics, 1997.

3 Khan KM, Bonar F, Desmond PM, et al. Patellar tendinosis (jumper's knee) findings at histopathologic examination, US, and MR imaging. Victorian Institute of Sport tendon Study Group. Radiology 1996;200:821-7.

4 Neuhold A, Stiskal M, Kainberger F, et al. Degenerative Achilles tendon disease: assessment by magnetic resonance and ultrasonography. Eur J Radiol 1992;14:213-20.

5 Khan KM, Cook JL, Bonar F, et al. Histopathology of common tendinopathies. Update and implications for clinical management. Sports Med 1999;27:393-408.

6 Movin T, Gad A, Reinholt FP. Tendon pathology in long-standing Achillodynia. Biopsy findings in 40 patients. Acta Orthop Scand 1997:68:170-5.

7 Alfredson H, Thorsen K, Lorentzon R. In situ microdialysis in tendon tissue: high levels of glutamate, but not prostaglandin $E_{2}$ in chronic Achilles tendon pain. Knee Surg Sports Traumatol Arthrosc 1999;7:378-81.

8 Alfredson $\mathrm{H}$, Pietilä $\mathrm{T}$, Jonsson $\mathrm{P}$, et al. Heavy-load eccentric calf muscle training for the treatment of chronic Achilles tendinosis. Am J Sports Med 1998;26:360-6.

9 Mafi N, Lorentson R, Alfredson H. Superior short-term results with eccentric calf muscle training compared to concentric training in a randomized prospective multicenter study on patients with chronic Achilles tendinosis. Knee Surg Sports Traumatol Arthrosc 2001;9:42-7.

10 Maffulli N, Regine R, Angelillo M, et al. Ultrasound diagnosis of Achilles tendon pathology in runners. Br J Sports Med 1987;21:158-62.

11 Paavola M, Paakkala T, Kannus P, et al. Ultrasonography in the differential diagnosis of Achilles tendon injuries and related disorders. Acta Radiol 1998;39:612-19.

12 Åström M, Gentz CF, Nilsson P, et al. Imaging in chronic Achilles tendinopathy: a comparison of ultrasonography, magnetic resonance imaging and surgical findings in 27 histologically verified cases. Skeletal Radiol 1996:l:615-20.

13 Weinberg EP, Adams MJ, Hollenberg GM. Color doppler sonography of patellar tendinosis. Am J Roentgenol 1998;171:743-44.

14 O'Reilly MA, Massouh H. Pictorial review: the sonographic diagnosis of pathology in the Achilles tendon. Clin Radiol 1993;48:202-6.

15 Alfredson H, Lorentzon R. Chronic Achilles tendinosis: recommendations for treatment and prevention. A review. Sports Med 2000;29:135-46.

16 Öhberg L, Lorentzon R, Alfredson H. Neovascularisation in Achilles tendons with painful tendinosis but not in normal tendons: an ultrasonographic investigation. Knee Surg Sports Traumatol Arthrosc 2001;9:233-8.
COMMENTARY

Achilles tendinosis has been linked to overuse stress, poor vascularity, lack of flexibility, genetic make up, sex, endocrine and metabolic factors, and the use of corticosteroids or quinolone antibiotics.

We do not know the origin of the pain in tendinopathies. Classically, pain has been attributed to inflammatory processes, but overuse tendinopathies are degenerative, not inflammatory conditions. Recently, the idea of a combination of mechanical and biochemical causes has become attractive. Tendon degeneration with mechanical breakdown of collagen could theoretically explain the pain mechanism. Also, high concentrations of the neurotransmitter glutamate have recently been found in patients with Achilles tendinopathy.

This investigation used high resolution real time ultrasonography to show that, in patients treated with eccentric calf muscle training, tendon thickness had decreased and tendon structure in the area with tendinosis had normalised when examined several years after the intervention. This article reports an association, but its design does not allow a definite cause-effect relation to be established. However, the idea that resolution of the clinical syndrome, with time, returns the tendon to its normal status does appeal to the scientific mind. We shall have to see whether these clinical and imaging results will remain stable with time, and whether they are applicable to the athletic population that remains active and wants to return to top level sports training and performance. Nevertheless, if it has worked in middle aged and older patients, it is promising for younger active patients!

Department of Trauma and Orthopaedic Surgery, Keele University School of Medicine, Thornburrow Drive, Hartshill ST4 7QB, UK; osal4@keele.ac.uk

Warm up

\section{Genedicine}

\section{P McCrory}

in ontinuing my occasional forays into gene technology, I was interested to see that regulatory authorities in China have licensed a gene therapy based treatment. This is the first time anywhere in the world that such a treatment has been authorized.

Although the therapy relates to head and neck squamous cell carcinoma (a huge problem in southern China) the technology is relatively straightforward. The company, SiBiono GeneTech, uses an adenovirus as the vector to insert a gene designed to limit cancer cell growth. The results of trials have been published in the peer reviewed Chinese Medical Journal and show this technique to be dramatically effective compared with conventional radiotherapy. The beauty of the technique is that the virus does not integrate into the cell genome (thereby minimising the risk of leukaemia) but rather confines itself to the malignant cell line. The simplicity of the technique is echoed in the low cost (about US\$ 360 per dose) and the ease by which virtually any doctor can administer the treatment.

Why is this of interest to practitioners of sports medicine? I have previously alluded to the issue of gene transfer technology as a threat to sports medicine. ${ }^{1}$ What we are seeing now is the transition of gene therapy from a promising experimental concept to an established mainstream treatment. While there is little doubt that gene medicine (or "genedicine") holds promise for the treatment of many currently untreatable diseases, it is also arguably the greatest threat facing modern sports medicine.
Imagine a simple and cheap treatment that can boost your red blood cell mass or give you bigger muscles on demand. Imagine also that this ergogenic therapy is undetectable. That is what genedicine is and can do at present-not in the future-now.

If you are an elite athlete and the Olympics or World Championships are coming up why consider an anabolic agent that can be picked up in drug tests or else needs a retinue of scientists manipulating the dose schedule to avoid detection? Once the bugs are sorted out, gene therapy is the way to go. The idea that scientists and government bodies will self regulate this area to prevent athletes accessing such treatments is simply ludicrous. They haven't done this with other ergogenic agents so why start here? The World AntiDoping Agency (www.wada-ama.org) seems to adhere to this belief. Good luck to them, but I seriously doubt whether anyone else in the world takes that view seriously.

Br J Sports Med 2004;38:11

\section{REFERENCE}

1 McCrory P. Super athletes of gene cheats? Br J Sports Med 2003;37:192-194. 